\title{
Identification of genes linked to gefitinib treatment in prostate cancer cell lines with or without resistance to androgen: A clue to application of gefitinib to hormone-resistant prostate cancer
}

\author{
SEIJI YANO ${ }^{1}$, HIDEYASU MATSUYAMA ${ }^{1}$, HIROSHI HIRATA ${ }^{1}$, RYO INOUE $^{1}$, HIROAKI MATSUMOTO $^{1}$, \\ CHIETAKA OHMI ${ }^{1}$, KOSHIRO MIURA ${ }^{2}$, MUTSUNORI SHIRAI ${ }^{2}$, NORIO IIZUKA ${ }^{3}$ and KATSUSUKE NAITO ${ }^{1}$
}

Departments of ${ }^{1}$ Urology, ${ }^{2}$ Microbiology, ${ }^{3}$ Surgery 2, Yamaguchi University

School of Medicine, 1-1-1 Minami-Kogushi, Ube, Yamaguchi 755-8505, Japan

Received January 10, 2006; Accepted February 17, 2006

\begin{abstract}
Understanding the molecular action of gefitinib, an epidermal growth factor receptor tyrosine kinase inhibitor, might allow us to perform more effective therapies for hormone-independent advanced prostate cancer. A DNA microarray study was undertaken to comprehensively analyze the alteration of levels of 1,081 genes after gefitinib treatment in androgen-independent PC3 and DU145 cells and androgen-dependent LNCaP cells. The proliferation of PC3, DU145 and LNCaP cells was significantly inhibited by $50.2 \%, 83.8 \%$ and $55.2 \%$, respectively, 6 days after $10 \mu \mathrm{M}$ gefitinib administration. Of the above 1,081 genes, we identified 23, 13 and 33 genes with significantly different expression in PC3, DU145 and LNCaP cells, respectively, $24 \mathrm{~h}$ after $10 \mu \mathrm{M}$-gefitinib exposure. Among the identified genes, only Quiescin Q6, a negative cell cycle regulator, was increased after gefitinib treatment in all three cell lines regardless of gefitinib sensitivity. Except for Quiescin Q6, there were no overlapping genes between PC3 and DU145 cells. However, levels of several oncogenes or proliferation-related genes were changed after gefitinib treatment in the 2 androgen-independent cell lines. We also identified 7 unique genes [ glycyl-tRNA synthetase, interferon, $\alpha$-inducible protein, stratifin, nuclear factor of $\kappa$ light polypeptide gene enhancer in B-cells 1, dual specificity phosphatase 9, guanine nucleotide binding protein ( $G$ protein) $\beta$ polypeptide 2 , neural retina leucine zipper] whose levels were altered exclusively after gefitinib administration in gefitinib-resistant PC3 and LNCaP cells, but not in DU145 cells, suggesting that these 7 genes could
\end{abstract}

Correspondence to: Dr Katsusuke Naito, Department of Urology, Yamaguchi University School of Medicine, 1-1-1 Minami-Kogushi, Ube, Yamaguchi 755-8505, Japan

E-mail: katsunai@po.cc.yamaguchi-u.ac.jp

Key words: gefitinib treatment, prostate cancer, androgen be targets for overcoming gefitinib resistance. Collectively, our molecular profiling data will serve as a framework for understanding the molecular action of gefitinib for prostate cancer.

\section{Introduction}

Prostate cancer, one of the common malignant tumors among older men, is usually treated by several therapeutic options, such as surgical or radiological procedures and androgendeprivation (hormone) therapy, according to the stage of tumor progression, patient age and physical status (1). Prostate cancer can be successfully managed by androgen-deprivation therapy when the tumor is in a hormone-sensitive status. Once the tumor becomes hormone-resistant, the median survival is only approximately 12 months, even when conventional therapeutic modalities are performed $(1,2)$. To improve the poor prognosis of this type of cancer, it is critical to understand the mechanism of the paradigm from sensitive to resistant to hormone therapy and to seek a novel treatment modality based on this mechanism.

It is well known that epidermal growth factor (EGF) is involved in the growth in many cancer cell types. Blockade of the EGF-mediated signal pathways drastically inhibits the proliferation of several cancer cells $(3,4)$, suggesting that blockade of EGF receptor (EGFR) may be useful in molecular target-based therapy. Interestingly, it has been reported that protein levels of EGFR increase during the natural history of prostate cancer and the progression from hormone-sensitive to resistant prostate cancers $(5,6)$. Gefitinib (ZD1839, Iressa ${ }^{\mathrm{TM}}$, AstraZeneca, Macclesfield, UK) is an orally active EGFR tyrosine kinase inhibitor that has shown anti-tumor activity in a broad range of human cancers including prostate cancer, either alone or in combination with other agents $(4,6-8)$. Gefitinib has been reported to inhibit EGFR tyrosine kinase and to show an anti-proliferative effect against both hormonesensitive and resistant prostate cancer in vivo and in vitro $(7,9)$, while the precise mechanism of blocking the EGFsignaling pathway has not fully been clarified (10-12). Accumulating evidence has indicated that inactivation of phosphatidylinositol 3-kinase (PI3K/Akt), which is located 


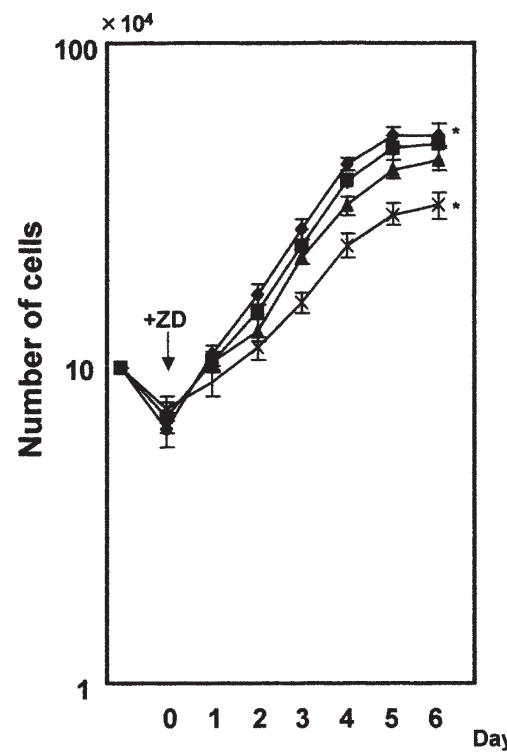

PC3

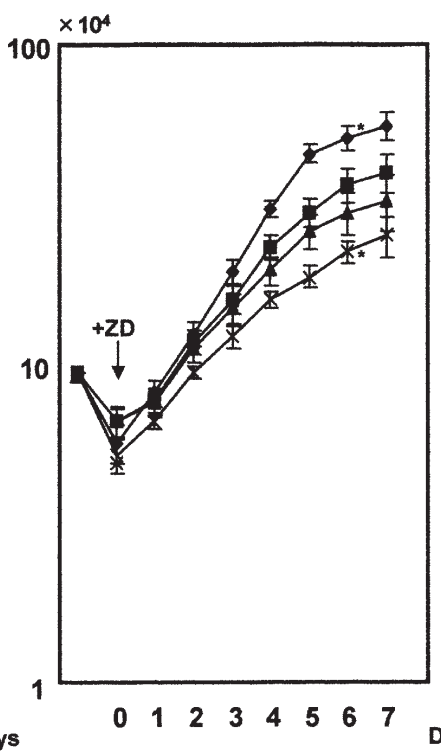

LNCaP

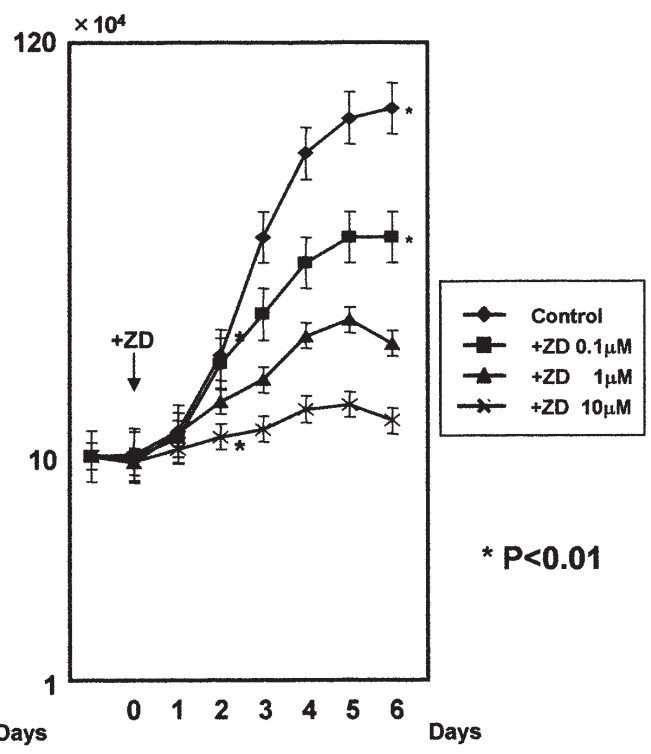

DU145

Figure 1. Growth curves of PC3, LNaP cells, and DU145 cells with and without gefitinib exposure. Statistically significant growth inhibition was observed in $\mathrm{PC} 3$, and LNCaP cells with $10 \mu \mathrm{M}$ gefitinib exposure at day 8 after seeding as compared with control ( $<<0.05$ ). Statistically significant growth inhibition was observed in DU145 cells with $0.1 \mu \mathrm{M}$ gefitinib exposure at day 8 after seeding as compared with control ( $\mathrm{p}<0.05)$. Bar indicates standard deviation (SD).

downstream of the EGFR pathway, may be lethal to several types of cancer cells (13-15). Phosphorylation of Akt (i.e. activation of $A k t)$ is inhibited by phosphatase and tensin homologue (PTEN) (16). To gain insights into the molecular basis underlying the blockade of the EGF-signaling pathway and to investigate the relation between EGF and hormonerelated signal pathways, we here evaluated the effects of gefitinib on the in vitro growth of a panel of prostate cancer cell lines with or without hormone-resistance and investigated genes whose expression differed between individual cell lines before and after drug administration using DNA microarray technology.

\section{Materials and methods}

Reagents and cell culture. Gefitinib was kindly provided by AstraZeneca. Stock solutions were prepared in dimethyl sulfoxide (DMSO), and stored at $-20^{\circ} \mathrm{C}$ until use. To minimize its cytotoxicity, the drugs were diluted in RPMI-1640 (Sigma Chemical Co., St. Louis, MO) immediately before use with final DMSO concentration of $<0.1 \%$. Among three established human prostate cancer cell lines, hormone-sensitive LNCaP cells and hormone-resistant PC-3 and DU-145 cells were used in this study. Cells were maintained in RPMI-1640 supplemented with $10 \%$ heat-inactivated fetal bovine serum (Gibco BRL, Grand Island, NY) and penicillin (100 UI/ml) in a humidified atmosphere of $95 \%$ air and $5 \% \mathrm{CO}_{2}$ at $37^{\circ} \mathrm{C}$.

Assessment of cell growth. The cells were plated at $1 \times 10^{5}$ per $3-\mathrm{cm}^{2}$ plate. After $24 \mathrm{~h}$, gefitinib was added to the culture medium at concentrations of $0.1,1$ and $10 \mu \mathrm{M}$ each, all of which are clinically achievable concentrations in the tissue. Viable cells were counted manually using the tripan blue dye exclusion test every $24 \mathrm{~h}$ for 8 days. Five independent counts were made from each condition. Mean counts were plotted against time, and the logarithmic tract of growth curves was made. After $72 \mathrm{~h}$ of plating, the percent inhibitory rate was calculated as compared with control. As a control, we used intact cells that were not exposed to gefitinib.

RNA extraction and preparation of probes. Prior to the DNA microarray study, we selected the $10 \mu \mathrm{M}$ concentration of gefitinib because it clearly discriminates sensitive from resistant cell lines. Total RNA was extracted from each cell line before and after 24-h incubation of $10 \mu \mathrm{M}$ gefitinib using the RNeasy kit (Qiagen, Valencia, CA) according to the manufacturer's instructions. Reverse transcription and fluorescence labeling were performed to synthesize fluorochrome-labeled cDNA probes with an RNA fluorescence labeling kit (BD Atlas PowerScript Fluorescent Labeling Kit; BD Biosciences, Palo Alto, CA) for cells with and without gefitinib treatment with Cy3-dUTP and Cy5-dUTP (Amersham Biosciences, Piscataway, NJ, USA), respectively, according to the manufacturer's recommendations.

DNA microarray. We used a commercially available DNA microarray kit (Atlas Glass Human 1.0 Microrray; BD Biosciences), which contained 1,081 clones. Labeled probes were mixed with a hybridization solution (GlassHyb Hybridization Solution; BD Biosciences). After hybridization for $16 \mathrm{~h}$ at $50^{\circ} \mathrm{C}$, the slides were washed in $1 \mathrm{X} \mathrm{SSC}$ and $0.1 \%$ SDS for $10 \mathrm{~min}$ at room temperature twice, and then $0.1 \mathrm{X}$ standard sodium citrate (SSC) and $0.1 \%$ sodium dodecyl sulfate (SDS) for $10 \mathrm{~min}$ at room temperature, and rinsed briefly with distilled water. The slides were scanned for $\mathrm{Cy} 3$ and $\mathrm{Cy} 5$ fluorescence with a fluoro-image analyzer (FLA-8000; Fuji film Co., Tokyo, Japan), and the fluorescence was quantified with analysis software (Array Gauge Ver.2.0; Fuji film Co.). 
Table I. Growth inhibition rate of each concentration at the 6th day after exposure to gefitinib.

\begin{tabular}{lccc}
\hline & \multicolumn{3}{c}{ Concentration of gefitinib $(\mu \mathrm{mol})$} \\
\cline { 2 - 4 } Cells & $0.1^{\mathrm{a}}$ & $1.0^{\mathrm{a}}$ & $10^{\mathrm{a}}$ \\
\hline PC3 $^{\mathrm{a}}$ & 16.9 & 33.5 & 50.2 \\
LNCaP $^{\mathrm{a}}$ & 29.8 & 49.5 & 55.2 \\
DU145 $^{\mathrm{a}}$ & 33.5 & 60.7 & $83.8 \%$ \\
\hline
\end{tabular}

${ }^{\mathrm{a}} \mathrm{P}<0.01$, ANOVA with Fisher's PLSD test.

Gene expression analysis. Fluorescence intensity measurements from each array element were compared to local background, and background subtraction was performed. To normalize the data, the Cy3 to $\mathrm{Cy} 5$ ratio for each gene was adjusted to a median ratio value of all spots in this array. Spots with signal intensity less than the mean value of negative control spots which is a peculiarity in this array were excluded from the following analysis. Poor hybridization spots or areas of the array with obvious defects were flagged manually. The Cy3 to Cy5 ratios in duplicate experiments were averaged in each spot. Genes were categorized as increased or decreased if the ratio difference was 4 -fold or greater.

Statistical analysis. The statistical analyses were performed using JMP Version 4.02 (SAS institute, Cary, NC). ANOVA with Fisher's PLSD test were used to compare differences in the number of viable cells among the three cell lines. $\mathrm{P}<0.01$ was considered significant.

\section{Results}

Growth inhibition of prostate cancer cell lines by gefitinib. Gefitinib inhibited the proliferation of the three cell lines in a dose-dependent manner (Fig. 1, Table I). The proliferation of PC3, DU145 and LNCaP cells was significantly inhibited by $50.2 \%, 83.8 \%$ and $55.2 \%$, respectively, 6 days after $10 \mu \mathrm{M}$ gefitinib administration (Table I, $\mathrm{P}<0.01$, ANOVA with Fisher's PLSD test).

The growth inhibition rate was significantly different among the three cell lines at each concentration 6 days after exposure (Table I, $\mathrm{P}<0.01$, ANOVA with Fisher's PLSD test). From these results, the sensitivity was tentatively ranked in order of DU145, LNCaP, and PC3. The proliferation of DU145 cells was inhibited significantly 2 days after $10 \mu \mathrm{M}$ gefitinib exposure. By contrast, the proliferation of the remaining 2 cell lines was not inhibited 2 days after $10 \mu \mathrm{M}$ gefitinib exposure (Fig. 1). This finding strikingly indicated that the anti-proliferative action by gefitinib can be observed within $48 \mathrm{~h}$ after administration; therefore, RNA sampling for the following DNA microarray analysis was performed before and $24 \mathrm{~h}$ after $10 \mu \mathrm{M}$-gefitinib exposure.

Genes altered after gefitinib treatment in PC-3 cells. We identified 23 genes with significantly different expression levels between PC-3 cells untreated and treated with gefitinib. In PC-3/gefitinib cells, 14 genes showed decreased levels by $>4$-fold, and 9 genes showed increased levels by $>4$-fold (Table II). The expression level of quiescin Q6, which is a negative cell cycle regulator gene, was markedly increased. On the other hand, guanylate cyclase and Stratifin, known as tumor suppressor genes, were markedly decreased.

Genes altered after gefitinib treatment in DU-145 cells. We identified 13 genes with significantly different expression levels between DU-145 cells untreated and treated with DU-145 gefitinib. In DU-145/gefitinib cells, 13 genes showed increased levels by $>4$-fold, but none showed decreased levels by $>4$-fold (Table III). In DU-145 cells, the expression level of quiescin $Q 6$ gene was markedly increased.

Genes altered after gefitinib treatment in LNCaP cells. We identified 33 genes with significantly different expression levels between LNCaP cells untreated and treated with gefitinib. In LNCaP/gefitinib cells, 12 genes showed decreased levels by $>4$-fold, and 21 genes showed increased levels by $>4$-fold (Table IV). In LNCaP cells, jagged 1, quiescin Q6 and glutathione $S$-transferase $\theta 1$ were markedly increased. The expression level of Stratifin was markedly decreased.

Genes altered exclusively in gefitinib-resistant PC3 and LNCaP cells. We highlighted the difference in gene expression patterns between gefitinib-resistant (LNCaP, PC3) cells and gefitinib-sensitive DU-145 cells. We identified 8 genes whose expression levels in PC-3/gefitinib and LNCaP/gefitinib cells were markedly different from those in DU-145/gefitinib cells. Among the 8 genes, one showed increased levels and 7 showed decreased levels in PC-3/gefitinib and LNCaP/gefitinib cells as compared with DU-145/gefitinib cells (Table V).

Involvement of PTEN-related genes in the antitumor activity of gefitinib. Based on the concept that PTEN pathway is involved in the EGF-relevant signaling or antitumor activity by gefitinib (17), we arbitrarily selected four PTEN-related genes from the 1081 genes in our array data (Table VI). The expression level of PTEN-related genes tended to be suppressed more strongly in DU145 cells than in PC3 and LNCaP cells after gefitinib treatment. The expression levels of phosphatidylinositol-4-phosphate 5-kinase, mouse double minute 2, known as negative regulator of p53 product, and $S H B$ adaptor protein were relatively decreased by approximately 2-fold in DU145/ gefitinib cells as compared with control.

\section{Discussion}

EGF and EGF receptor (EGFR) play an important role in the pathogenesis of a variety of tumors, representing a promising therapeutic target (18). It was reported that the protein levels of both EGF and EGFR were higher in prostate cancer than in non-cancerous prostate $(19,20)$. Notably, a change from paracrine to autocrine EGFR regulation has been found in hormone-resistant prostate cancer (5). On the basis of these findings, it is not difficult to assume that the EGF-EGFR pathway activated by prostate cancer cells is closely related to their higher malignant potential and could explain the more advanced stage of this malignancy (5). From this standpoint, a 
Table II. Genes whose levels were altered in PCS cells after exposure to gefitinib.

A. Increased genes

No.

Gene name

Ratio

\begin{tabular}{lll}
\hline 1 & quiescin $06^{b}$ & $3.82^{\mathrm{b}}$ \\
2 & fibroblast growth factor $6^{\mathrm{b}}$ & 3.25 \\
3 & erythropoietin receptor & 3.19 \\
4 & replication protein Al $(70 \mathrm{kDa})$ & 2.80 \\
5 & growth arrest and DNA-damage-inducible, $\alpha$ & 2.66 \\
6 & cell growth regulatory with ring finger domain & 2.48 \\
7 & epidermal growth factor receptor pathway substrate 15 & 2.29 \\
8 & v-ski avian sarcoma viral oncogene homolog & 2.12 \\
9 & glycyl-tRNA synthetase & $2.11^{\mathrm{a}}$ \\
\hline
\end{tabular}

B. Decreased genes

No.

Gene name

Ratio

\begin{tabular}{|c|c|c|}
\hline 1 & guanylate cyclase $2 D$, membrane (retina-specific) & -3.27 \\
\hline 2 & Stratifin $^{\mathrm{a}}$ & $-3.16^{\mathrm{a}}$ \\
\hline 3 & muti. (E. coli) homolog 1 (colon cancer, nonpolyposis type 2) & -2.51 \\
\hline 4 & neuronal pentraxin II & -2.47 \\
\hline 5 & leptin receptor & -2.45 \\
\hline 6 & cytochrome P450, subfamily IIF, polypeptide 1 & -2.39 \\
\hline 7 & adenosine Al receptor & -2.37 \\
\hline 8 & nuclear factor of $\kappa$ light polypeptide gene enhancer in B-cells $1(p 105)^{\mathrm{a}}$ & $-2.32^{\mathrm{a}}$ \\
\hline 9 & growth arrest and DNA-damage-inducible, $\gamma$ & -2.31 \\
\hline 10 & interferon, $\alpha$-inducible protein (clone IFI-6-16) ${ }^{\mathrm{a}}$ & $-2.29^{\mathrm{a}}$ \\
\hline 11 & tumor necrosis factor receptor superfamily, member $1 B$ & -2.19 \\
\hline 12 & dual specificity phosphatase $9^{\mathrm{a}}$ & $-2.18^{\mathrm{a}}$ \\
\hline 13 & neural retina leucine zipper ${ }^{\mathrm{a}}$ & $-2.13^{\mathrm{a}}$ \\
\hline 4 & guanine nucleotide binding protein $(G$ protein $), \beta$ polypeptide $2^{\mathrm{a}}$ & $-2.05^{\mathrm{a}}$ \\
\hline
\end{tabular}

Ratio: $\log 2\left(\right.$ cy3/cy5). ${ }^{\mathrm{a} C o i n c i d e n t ~ w i t h ~ L N C a P . ~}{ }^{\mathrm{b} C o i n c i d e n t ~ w i t h ~ a n o t h e r ~ t w o ~ c e l l ~ l i n e s . ~}$

Table III. Genes whose levels were increased in DU-145 cells after exposure to gefitinib.

\begin{tabular}{rlr}
\hline No. & \multicolumn{1}{c}{ Gene name } & Ratio \\
\hline 1 & mutated in colorectal cancers & 2.93 \\
2 & cystic fibrosis transmembrane conductance regulator & 2.62 \\
3 & quiescin Q6 & $2.39^{\mathrm{a}}$ \\
4 & v-myc avian myelocytomatosis viral oncogene homolog & 2.35 \\
5 & serine (or cysteine) proteinase inhibitor & 2.31 \\
6 & glial cell derived neurotrophic factor & 2.26 \\
7 & POU domain, class 5, transcription factor 1 & 2.26 \\
8 & neurotensin & 2.21 \\
9 & breast cancer 2, early onset & 2.19 \\
10 & C-reactive protein, pentraxin-related & 2.13 \\
11 & interferon $\gamma$ receptor 2 & 2.07 \\
13 & adenylate cyclase 6 & 2.05 \\
\hline
\end{tabular}

Ratio: $\log 2$ (cy3/cy5). ${ }^{\mathrm{a} C o i n c i d e n t}$ with another two cell lines. 
Table IV. Genes whose levels were altered in LNCaP cells after exposure to gefitinib.

A. Increased genes

No.

Gene name

Ratio $^{\mathrm{a}}$

\begin{tabular}{|c|c|c|}
\hline 1 & jagged 1 (Alagille syndrome) & 6.10 \\
\hline 2 & quiescin $06^{\mathrm{b}}$ & $3.64^{\mathrm{b}}$ \\
\hline 3 & glutathione S-transferase $\theta 1$ & 3.07 \\
\hline 4 & Ran GTPase activating protein 1 & 2.99 \\
\hline 5 & growth factor receptor-bound protein 10 & 2.99 \\
\hline 6 & superoxide dismutase 1, soluble [amyotrophic lateral sclerosis 1 (adult)] & 2.61 \\
\hline 7 & small inducible cytokine B subfamily (Cys-X-Cys motif), member 13 (B-cell chemoattractant) & 2.58 \\
\hline 8 & cyclin 1 & 2.45 \\
\hline 9 & ras homolog gene family, member $E$ & 2.37 \\
\hline 10 & mitogen-activated protein kinase 6 & 2.32 \\
\hline 11 & Vertebrate LIN7 homolog 1, Tax interaction protein 33 & 2.28 \\
\hline 12 & colony stimulating factor 3 (granulocyte) & 2.23 \\
\hline 13 & CDC6 (cell division cycle 6, S. cerevisiae) homolog & 2.21 \\
\hline 14 & v-myc avian myelocytomatosis viral oncogene homolog & 2.21 \\
\hline 15 & v-abl Abelson murine leukemia viral oncogene homolog 1 & 2.20 \\
\hline 16 & BRCAl associated RING domain 1 & 2.20 \\
\hline 17 & integrin, $\beta 5$ & 2.16 \\
\hline 18 & Purkinje cell protein 4 & 2.14 \\
\hline 19 & glycyl-tRNA synthetase $e^{\mathrm{a}}$ & $2.09^{\mathrm{a}}$ \\
\hline 20 & SH3-domain binding protein 2 & 2.05 \\
\hline 21 & ADP-ribosyltransferase [NAD+; poly (ADP-ribose) polymerase] & 2.04 \\
\hline
\end{tabular}

\section{B. Decreased genes}

No.

Gene name

Ratio

\begin{tabular}{|c|c|c|}
\hline 1 & interferon, $\alpha$-inducible protein (clone IFI-6-16) ${ }^{\mathrm{a}}$ & $-2.81^{\mathrm{a}}$ \\
\hline 2 & Stratifin ${ }^{\mathrm{a}}$ & $-2.76^{\mathrm{a}}$ \\
\hline 3 & low density lipoprotein-related protein-associated protein 1 & -2.59 \\
\hline 4 & neural precursor cell expressed, developmentally down-regulated 5 & -2.55 \\
\hline 5 & matrix metalloproteinase 17 (membrane-inserted) & -2.49 \\
\hline 6 & nuclear factor of $\kappa$ light polypeptide gene enhancer in B-cells $1(p 105)^{\mathrm{a}}$ & $-2.43^{\mathrm{a}}$ \\
\hline 7 & dual specificity phosphatase $9^{a}$ & $-2.34^{\mathrm{a}}$ \\
\hline 8 & death associated protein 3 & -2.31 \\
\hline 9 & guanine nucleotide binding protein $(G$ protein $), \beta$ polypeptide $2^{\mathrm{a}}$ & $-2.27^{\mathrm{a}}$ \\
\hline 10 & ubiquitin-conjugating enzyme E2A & -2.14 \\
\hline 11 & interleukin $12 \mathrm{~A}$ & -2.10 \\
\hline 12 & neural retina leucine zipper ${ }^{\mathrm{a}}$ & $-2.06^{\mathrm{a}}$ \\
\hline
\end{tabular}

Ratio: $\log 2$ (cy3/cy5). ${ }^{\mathrm{a} C o i n c i d e n t ~ w i t h ~ P C-3 . ~}{ }^{\mathrm{b}}$ Coincident with another two cell lines.

possible modulation of the EGF-relevant pathway is very attractive and may provide new therapeutic options against hormone-resistant prostate cancer at an advanced stage. Although much effort has been devoted to elucidating the signal transduction pathway of EGFR in human prostate cancer (10-12), the molecular basis has not yet been fully disclosed. To the best of our knowledge, our current study is the first to comprehensively analyze the alteration of genes by gefitinib in prostate cancer cells for association with hormone (i.e., androgen) resistance and provides a clue to the application of gefitinib in the treatment of hormone-resistant advanced prostate cancer. In the present study, gefitinib showed significantly higher growth inhibition of DU-145 cells than of the other two cell lines. This result is reasonable considering that the PTEN gene was intact in DU-145 cells, but not in PC3 and LNCaP cells $(21,22)$.

In addition to the finding of the PTEN status, we found that the expression level of quiescin Q6 gene was uniformly 
Table V. Genes altered exclusively in gefitinib-resistant PCS and LNCap cells.

\begin{tabular}{|c|c|c|c|c|}
\hline No. & Gene name & Ratio $/$ PC3 & $/ \mathrm{LNCaP}$ & /DU145 \\
\hline I & glycyl-tRNA synthetase & $2.12 \Delta$ & $2.09 \boldsymbol{\Delta}$ & 0.13 \\
\hline 2 & interferon, $\alpha$-inducible protein (clone IFI-6-16) & $-2.29 \nabla$ & $-2.81 \nabla$ & -0.15 \\
\hline 3 & stratifin & $-3.17 \nabla$ & $-2.77 \nabla$ & -0.68 \\
\hline 4 & nuclear factor of $\kappa$ light polypeptide gene enhancer in B-cells 1 & $-2.32 \nabla$ & $-2.43 \nabla$ & -0.22 \\
\hline 5 & dual specificity phosphatase 9 & $-2.19 \nabla$ & $-2.34 \nabla$ & 0.63 \\
\hline 6 & guanine nucleotide binding protein ( $G$ protein), $\beta$ polypeptide 2 & $-2.05 \nabla$ & $-2.28 \nabla$ & -0.53 \\
\hline 7 & neural retina leucine zipper & $-2.13 \nabla$ & $-2.06 \nabla$ & -0.22 \\
\hline
\end{tabular}

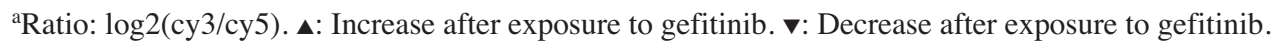

Table VI. PTEN-related genes after gefitinib treatment in PC3, LNCap and DU145 cells.

\begin{tabular}{|c|c|c|c|c|}
\hline No. & Gene name & Ratio $/ \mathrm{PC} 3$ & $/ \mathrm{LNCaP}$ & /DU145 \\
\hline 1 & phosphatidylinositol-4-phosphate 5-kinase, type I & -0.22 & -0.81 & -1.05 \\
\hline 2 & phosphatidylinositol-4-phosphate 5-kinase, type 11 & 0.16 & -0.19 & -1.11 \\
\hline 3 & mouse double minute 2 & -0.15 & -0.38 & -1.28 \\
\hline 4 & SHB adaptor protein & -0.11 & -1.01 & -1.19 \\
\hline
\end{tabular}

Ratio: $\log 2(\operatorname{cy} 3 /$ cy5).

increased in all three cell lines after gefitinib treatment. Quiescin Q6 has been reported to act as a negative cell cycle regulator and inhibits the cell proliferation control in other cancers, such as breast and liver cancers $(23,24)$. Our data showing significant growth inhibition in three cell lines at a $10 \mu \mathrm{M}$ gefitinib concentration suggest that Quiescin Q6 plays a distinct role from the PTEN pathway in the anti-proliferative effect of gefitinib in prostate cancer. We, therefore, suggest that the quiescin $Q 6$ gene can be a potent candidate for the modulation of anti-tumor activity of gefitinib.

The expression level of the glycyl-tRNA synthetase gene was significantly increased by gefitinib treatment in $\mathrm{PC} 3$ and LNCaP cells in comparison with DU145 cells. Although Glycyl-tRNA, an aminoacyl-tRNA, has been reported to be highly expressed in thyroid and liver cancer cells $(25,26)$, there are no studies reporting the alteration of this gene in prostate cancer. In hepatocellular carcinoma, upregulation of glycyltRNA synthetase was induced by low oxygen response in association with erythropoietin gene expression (26). Notably, in accordance with this result (26), we observed that both glycyl-tRNA synthetase and erythropoietin receptor were upregulated by gefitinib treatment in the PC 3 cell line, suggesting that the alteration of glycyl-tRNA synthetase can account for the low oxygen state in the cell caused by gefitinib.

Among the other six genes whose alteration was specific to DU-145 cells, stratifin was strongly downregulated in PC3 as well as LNCaP after gefitinib treatment. The stratifin/14-3-3sigma gene is involved in cell-cycle control in a p53-dependent manner, and prevents the accumulation of chromosomal damage. This result may be supported by the previous report that frequent epigenetic silencing of the gene has been reported in several types of cancer, including prostate, suggesting that the loss of stratifin expression may be causally involved in tumor progression (27).

Nagano et al reported that crosstalk between the EGF- and IFN-induced signalling pathways and the regulation of IFNinducible genes are altered in prostate cancer cells (28). STAT proteins are a group of latent cytoplasmic transcription factors which function as signal transducers and activators of transcription. Stat1 and -2 were originally identified to function in interferon signaling, and Stat1 was also found to be activated by epidermal growth factor (EGF) and other cytokines (29). Zhang et al reported that inhibition of cell proliferation by EGFR-related protein was accompanied by a concomitant attenuation of activation of mitogen-activated protein (MAP) kinases, AKT, and NF-кB (30), suggesting the identical attenuation of NF- $\mathrm{NB}$ by gefitinib treatment. Down regulation of both IFN $\alpha$ inducible genes and NF- $\kappa \mathrm{B}$ is likely to be due to either direct, or crosstalk suppression of EGFR by gefitinib, although this pathway has a minor role in the anti-proliferative effect in prostate cancer cell lines.

dual-specificity phosphatase 9, also known as MKP-4 (mitogen-activated protein kinase phosphatase 4) has a crucial role in cell cycle progression, and interacts with EGFR. MAPK-4 attenuates p38 MAPK during dexamethasoneinduced insulin resistance in 3T3-L1 adipocytes (31). Downregulation of MKP-4 may induce up-regulation of p38 MAPK, which is consistent with up-regulation of MAPK-6 in LNCaP in our study. Cross-communication between the Met 
receptor tyrosine kinase and EGFR has been proposed to involve direct association of both receptors and EGFR kinase-dependent phosphorylation. Cell surface receptor cross-talk involving the Met receptor, G protein-coupled receptors, and EGFR to the oncogenic potential of Met signaling was reported in human carcinoma cells (32). Neural retina specific gene $n r l$ encodes members of a new bZIP protein subfamily. Nrl has dimerization and DNA binding properties with its leucine zipper motif, and was also able to form heterodimers with Fos and Jun in vitro (33). All genes depicted in Table V were deeply involved in the EGFRrelated signal pathway, or crosstalk to other receptors, although the actual role of altered expression in each gene remains to be elucidated.

We identified the molecular signatures of prostate cancer cells in vitro after gefitinib administration in relation to hormone dependency. In this viewpoint, there may be a limitation of our present study in understanding the development of prostate cancer. However, we took clinically achievable concentration into consideration, supporting the concept that the condition used here could be close to the in vivo situation of prostate cancer and our identified genes might be related to the context of this cancer when gefitinib is clinically used.

In conclusion, gefitinib has an anti-tumor effect on prostate cancer cell lines in vitro. A panel of genes including quiescin $Q 6$, or stratifin identified in this study and the expression status of PTEN may aid the selection of patients who could benefit from gefitinib, from among those with hormone-resistant prostate cancer. Further studies are needed to investigate how these identified genes are involved in the biological action of gefitinib and to evaluate the efficacy of these genes as molecular targets combined with gefitinib.

\section{Acknowledgements}

This study was supported by a Grant-in-Aid for Science Research (no. 14370513) from Japan Society for the Promotion of Science (JSPS). The authors thank the AstraZeneca group of companies for providing gefitinib ("Iressa", ZD1839). "Iressa" is a trademark of the AstraZeneca group of companies.

\section{References}

1. Boyle P and Severi G: Epidemiology of prostate cancer chemoprevention. Eur Urol 35: 370-376, 1999.

2. Rambeaud JJ: Intermittent complete androgen blockade in metastatic prostate cancer. Eur Urol 35 (suppl 1): 32-36, 1999.

3. Woodburn $\mathbf{J}$ : The epidermal growth factor receptor and its inhibition in cancer therapy. Pharmacol Ther 82: 241-250, 1999.

4. Ciardiello F and Tortora F: A novel approach in the treatment of cancer: Targeting the epidermal growth factor receptor. Clin Cancer Res 7: 2958-2970, 2001.

5. Sher HI, Sarkis A, Reuter V, et al: Changing pattern of expression of the epidermal growth factor receptor and transforming growth factor in the progression of prostatic neoplasms. Clin Cancer Res 1: 545-550, 1995.

6. Barton J, Blackledge $G$ and Wakeling A: Growth factors and their receptors: new targets for prostate cancer therapy. Urology 58: 114-122, 2001.

7. Sirotnak FM, She Y, Lee F, Chen J and Scher HI: Studies with CWR22 xenografts in nude mice suggest that ZD1839 may have a role in the treatment of both androgen-dependent and androgen-independent human prostate cancer. Clin Cancer Res 8: 3870-3876, 2002.
8. Ciardiello F, Caputo R, Bianco R, et al: Inhibition of growth factors production and angiogenesis in human cancer cells by ZD1839 (Iressa), a selective epidermal growth factor receptor tyrosine kinase inhibitor. Clin Cancer Res 7: 1459-1465, 2001.

9. Vicentini C, Festuccia C, Gravina GL, Angelucci A, Marronaro A and Bologna M: Prostate cancer cell proliferation is strongly reduced by the epidermal growth factor receptor tyrosine kinase inhibitor ZD1839 in vitro on human cell lines and primary cultures. J Cancer Res Clin Oncol 129: 165-174, 2003.

10. Bianco R, Caputo R, Damiano V, et al: Combined targeting of epidermal growth factor receptor and MDM2 by gefitinib and antisense MDM2 cooperatively inhibit hormone-independent prostate cancer. Clin Cancer Res 10: 4858-4864, 2004.

11. Banaccorsi L, Marchiani S, Moratori M, et al: Gefitinib ('Iressa', ZD1839) inhibits EGF-induced invasion in prostate cancer cells by suppressing PI3K/AKT activation. J Cancer Res Clin Oncol 130: 604-614, 2004.

12. Sgambato A, Camerini A, Faraglia B, et al: Targeted inhibition of the epidermal growth factor receptor-tyrosine kinase by ZD1839 ('Iressa') induced cell-cycle arrest and inhibits proliferation in prostate cancer cells. J Cell Physiol 201: 97-105, 2004.

13. Cappuzo F, Magrini E, Ceresoli GL, et al: Akt phosphorylation and gefitinib efficacy in patients with advanced non-small-cell lung cancer. J Natl Cancer Inst 96: 1133-1141, 2004.

14. Moasser MM, Basso A, Averbuch SD and Rosen N: The tyrosine kinase inhibitor ZD1839 ('Iressa') inhibits HER2-driven signaling and suppresses the growth of HER2-overexpressing tumor cells. Cancer Res 61: 7184-7188, 2001

15. Bianco R, Shin I, Ritter CA, et al: Loss of PTEN/MMAC1/ TEP in EGF receptor-expressing tumor cells counteracts the antitumor action of EGFR tyrosine kinase inhibitors. Oncogene 22: 2812-2822, 2003.

16. Cantley LC: The phosphoinositide 3-kinase pathway. Science 296: 1655-1657, 2002.

17. Kokubo Y, Gemma A, Noro R, et al: Reduction of PTEN protein and loss of epidermal growth factor receptor gene mutation in lung cancer with natural resistance to gefitinib (IRESSA). Br J Cancer 92: 1711-1719, 2005.

18. Normanno N, Bianco C, De Luca, Maiello MR and Salmon DS: Target-based agents against ErbB receptors and their ligands: a novel approach to cancer treatment. Endocr-Relat Cancer 10: 1-21, 2003.

19. Russell PJ, Bennett S and Stricker P: Growth factor involvement in progression of prostate cancer. Clin Chem 44: 705-723, 1998.

20. Di Lorenzo G, Tortora G, D'Armient FP, et al: Expression of epidermal growth factor receptor correlates with disease relapse and progression to androgen-independence in human prostate cancer. Clin Cancer Res 8: 3438-3444, 2002.

21. Bistola DR, Pahwa GS, Lin MF and Cheng PW: Downregulation of PTEN/MMAC/TEP1 expression in human prostate cancer cell line DU145 by growth stimuli. Mol Cell Biochem 236: 75-81, 2002.

22. Huang H, Cheville JC, Pan Y, et al: PTEN induces chemosensitivity in PTEN-mutated prostate cancer cell by suppression of Bcl-2 expression. J Biol Chem 276: 38830-38836, 2001.

23. Moggs JG, Murphy TC, Lim FL, et al: Anti-proliferative effect of estrogen in breast cancer cells that re-express ER \{alpha\} is mediated by aberrant regulation of cell cycle genes. J Mol Endocrinol 34: 535-551, 2005.

24. Chiba T, Yokosaku O, Fukai K, et al: Cell growth inhibition and gene expression induced by the histone deacetylase inhibitor, trichostatin A, on human hepatoma cells. Oncology 66: 481-491, 2004.

25. Wasenius VM, Hemmer S, Kettunen E, et al: Hepatocyte growth factor receptor, metalloproteinase-11, tissue inhibitor of metalloproteinase-1, and fibronectin are up-regulated in papillary thyroid carcinoma: a cDNA and tissue microarray study. Clin Cancer Res 9: 68-75, 2003.

26. Scandurro AB, Weldon CW, Figueroa YG, et al: Gene microarray analysis reveals a novel hypoxia signal transduction pathway in human hepatocellular carcinoma analysis. Int J Oncol 19: 129-135, 2001.

27. Lodygin $\mathrm{D}$ and Hermeking $\mathrm{H}$ : The role of epigenetic inactivation of 14-3-3sigma in human cancer. Cell Res 15: 237-246, 2005.

28. Nagano K, Masters JR, Akpan A, Yang A, Corless S, Wood C, Hastie C, Zvelebil M, Cramer R and Naaby-Hansen S: Differential protein synthesis and expression levels in normal and neoplastic human prostate cells and their regulation by type I and II interferons. Oncogene 23: 1693-1703, 2004. 
29. Cao X, Tay A, Guy GR and Tan YH: Activation and association of Stat3 with Src in v-Src-transformed cell lines. Mol Cell Biol 16: 1595-1603, 1996.

30. Zhang Y, Banerjee S, Wang ZW, Marciniak DJ, Majumdar AP and Sarkar FH: Epidermal growth factor receptor-related protein inhibits cell growth and induces apoptosis of $\mathrm{BxPC} 3$ pancreatic cancer cells. Cancer Res 65: 3877-3882, 2005.

31. Bazuine M, Carlotti F, Tafrechi RS, Hoeben RC and Maassen JA: Mitogen-activated protein kinase (MAPK) phosphatase- 1 and -4 attenuate p38 MAPK during dexamethasone-induced insulin resistance in 3T3-L1 adipocytes. Mol Endocrinol 18: 1697-1707, 2004.
32. Fischer OM, Giordano S, Comoglio PM and Ullrich A: Reactive oxygen species mediate Met receptor transactivation by $\mathrm{G}$ protein-coupled receptors and the epidermal growth factor receptor in human carcinoma cells. J Biol Chem 279: 2897028978, 2004.

33. Kerppola TK and Curran T: Maf and Nrl can bind to AP-1 sites and form heterodimers with Fos and Jun. Oncogene 9: 675-684, 1994. 